Economía, Sociedad y Territorio, vol. vIII, núm. 27, 2008, 565-585

\title{
Indicadores sintéticos: una revisión de los métodos de agregación
}

\section{Synthetic indicators: a revision of aggregation methods}

\author{
JosÉ MONDÉJAR-JIMÉNEZ \\ Manuel Vargas-Vargas"
}

\begin{abstract}
An adequate way to objectively monitor the current economical situation consists on applying statistical/econometric models. Several difficulties may arise from the available indicators used; however these can be avoided by using synthetic indicators. In order to do so, it is necessary to select basic economic indicators, extract from them relevant information and aggregate them in a convenient way to obtain a synthetic indicator that summarises their common characteristics. In this paper we analyse several procedures and techniques to create traditional synthetic indicators; we also include new formulations based on indicator aggregation in the state space.
\end{abstract}

Keywords: synthetic indicators, economical analysis, aggregation methods.

\section{Resumen}

Una vía adecuada para objetivar el seguimiento económico coyuntural consiste en recurrir a los modelos estadístico-econométricos. Sin embargo, a través de los indicadores disponibles dicho seguimiento presenta diversas dificultades que se pueden evitar si se recurre a la elaboración de un indicador sintético. Para ello es necesario elegir los indicadores económicos básicos, extraer su información relevante y agregarlos convenientemente para elaborar un indicador sintético que resuma las características comunes a todos ellos. En el presente trabajo se analizan diversos procedimientos y técnicas para la elaboración de indicadores sintéticos tradicionales, así como la incorporación de nuevas formulaciones basadas en la agregación de indicadores en espacio de estados.

Palabras clave: indicadores sintéticos, análisis económico y métodos de agregación.

*Universidad de Castilla-La Mancha, España. Correos-e: Jose.Mondejar@uclm.es, Manuel.Vargas@uclm.es. 


\section{Introducción ${ }^{1}$}

La medición del desarrollo económico y la definición de los factores condicionantes del crecimiento son dos de las cuestiones de mayor interés en las últimas décadas; por ello, han centrado el interés de los científicos en general y de los economistas en particular. Dentro de este nivel de estudio nos centraremos en el análisis coyuntural de las macromagnitudes económicas.

En la actualidad, en el análisis coyuntural regional se pueden distinguir tres niveles de actuación para valorar e interpretar correctamente los datos estadísticos con el fin último de estudiar la evolución de la actividad económica.

1. Análisis a través de indicadores simples.

2. Análisis a través de indicadores sintéticos o complejos.

3. Estimación de la contabilidad trimestral regional.

Un esquema del proceso desarrollado en este trabajo y que corresponde con la construcción de indicadores sintéticos de actividad puede resultar como sigue:

- Selección de indicadores parciales.

- Tratamiento estadístico previo.

- Extracción de señal ciclo-tendencia y desestacionalización.

- Agregación de indicadores parciales.

- Indicador sintético.

Aquí podemos observar los distintos pasos previos a la agregación de los indicadores parciales. Aunque el objetivo de este trabajo es exponer los diferentes métodos de agregación (desarrollados en el apartado 1), realizaremos un breve repaso de las fases anteriores.

El primer paso de este proceso consiste en hacer una selección de las series de indicadores que se van a tomar en consideración. Sólo incluimos los indicadores cualitativos porque son los más recomendados para el seguimiento de la coyuntura económica. La selección de estas series se debe hacer de acuerdo con ciertos criterios formales como la disponibilidad de los datos, su significado económico, el tipo de frecuencia, etcétera.

${ }^{1}$ Los autores desean dejar constancia de su agradecimiento a los evaluadores, cuyos comentarios y sugerencias contribuyeron a mejorar sensiblemente este trabajo. 
Una vez que se seleccionaron los indicadores simples, es necesario -en la etapa preliminar- realizar un tratamiento previo de las series que consiste en tomar en cuenta aspectos estadísticos y económicos que requieren un tratamiento elemental, como deflactar el indicador simple, eliminar el efecto calendario, datos atípicos (outliers), fechado (siempre que sea posible) y estructura del retardo temporal.

Además, existe la necesidad de trabajar con series ajustadas de estacionalidad y componentes irregulares, es decir, se trata de obtener indicadores que no contengan ruido. Para ello es preciso tratar estadísticamente y filtrar las series con el fin de extraer la señal ciclo-tendencia. Este proceso de filtrado imposibilita el trabajo con indicadores cualitativos, aunque si bien éstos también podrían utilizarse con los diferentes métodos de agregación que se proponen a continuación, por lo que no deben mezclarse con los indicadores cuantitativos.

Un filtro ideal es aquél que está diseñado para permitir el paso de información en una banda de frecuencias y eliminar o inhibir la información contenida en otras bandas consideradas no deseables. Se trata de analizar qué tipo de frecuencia deja pasar cada filtro y cuáles de ellas son eliminadas, así como la eficacia relativa de cada filtro respecto de las frecuencias filtradas. Cualquier filtro tiene un coste informativo que se traduce en las observaciones que se pierden en el tramo final e inicial. Los métodos más importantes y usuales son filtros autorregresivos (AR), los filtros aditivos o de medias móviles (MA), el filtro bayesiano en espacio de estados, la extracción de la señal relevante con Seats (Maravall, 1989), entre otros.

\section{Métodos de agregación}

Una vez concluida la fase de filtrado, pasamos a la agregación de los indicadores parciales para obtener el indicador sintético. Se denomina indicador sintético a cualquier combinación de indicadores individuales, a los cuales nos referimos como indicadores componentes (Cabrer et al. 2001). Los indicadores sintéticos están sujetos a diversas críticas. La principal es que constituyen una aproximación puramente empírica al problema de la medición del nivel y el ritmo de la actividad económica. Entre sus ventajas destaca su sencillez, la cual en términos de complejidad teórica y de información necesaria permite realizar estimaciones 
y predicciones, además de la prontitud con la que se obtienen los resultados en comparación con otros métodos alternativos.

La mayor dificultad -y requisito imprescindible para la construcción de un indicador sintético regional de coyuntura- es la necesidad de disponer de una amplia base de datos de índole regional y de periodicidad mensual o trimestral (indicadores parciales) que permitan captar las fluctuaciones periódicas de corto plazo de la economía objeto de análisis. A continuación exponemos los métodos de agregación tradicionales.

\subsection{Métodos simples}

Un indicador compuesto de actividad se obtiene mediante la suma ponderada de los cambios en las series económicas individuales

$$
I C A=\sum_{i=1}^{n} w \cdot x \cdot x
$$

donde $I C A_{t}$ es el indicador compuesto en el periodo $t$, $x_{i t}$ es el valor del indicador $i$ en el periodo $t, n$ es el número de indicadores utilizados y $w_{i}$ es la ponderación asignada a cada uno de los indicadores parciales que, como es lógico, se debe cumplir

$$
\sum_{i=1}^{n} w_{i}=1
$$

Un indicador sintético consiste, por tanto, en una serie indexada que engloba y agrega la información contenida en un conjunto de indicadores parciales representativos de los diferentes sectores de la economía analizada. El principal problema que se presenta es el de la definición de los coeficientes de ponderación y la heterogeneidad en las unidades de los indicadores parciales.

El primer criterio que se utiliza para asignar una ponderación a las diferentes series que forman el indicador compuesto es otorgar a cada una de ellas el mismo peso, es decir, en este caso todas las series tienen la misma importancia en la construcción del indicador compuesto. Si se seleccionan $n$ indicadores para su construcción, la agregación de cada uno de ellos en la elaboración del indicador compuestos será

$$
w=\frac{1}{n}, \text { donde } i=1,2, \ldots, n
$$


Un segundo criterio para seleccionar el peso de cada uno de los indicadores parciales se basa en un criterio ad-hoc, el investigador fija a priori la importancia que tiene cada uno de los indicadores para la elaboración del indicador compuesto, en función de la importancia que tengan las distintas ramas o sectores en relación con el indicador de referencia. El conocimiento de la actividad económica por parte del analista juega aquí un papel quizá más importante, si cabe, puesto que una mala asignación $a$ priori de las ponderaciones puede conllevar un incremento del nivel de error cometido. No obstante, este método se puede perfeccionar teniendo en cuenta la volatilidad de cada uno de los indicadores parciales que forman parte del indicador compuesto, para ello debemos calcular la desviación estándar de cada uno de los indicadores parciales, así como su inversa.

De este modo, el índice compuesto se calcula a partir de la siguiente expresión:

$$
I C A_{t}=\sum_{i=1}^{n} w_{i} \cdot S_{i}^{*} \cdot x_{i t}
$$

donde wi y xit tienen el mismo significado que en la fórmula considerada en el apartado anterior y $\mathrm{Si}^{*}$ es la inversa de la desviación estándar de cada uno de los indicadores parciales. Por último, obtendremos el peso de cada uno de los indicadores simples como el producto de los pesos individuales por la inversa de la desviación estándar, y haciendo que la suma de los mismos sea unitaria (Pons, 1995).

Un tercer criterio para seleccionar los pesos de cada uno de los indicadores se basa en el análisis de la correlación entre cada uno de éstos y una variable que registra la evolución del conjunto de la actividad económica, como puede ser, por ejemplo, el valor añadido bruto (VAB) o el producto interno bruto (PIB), ya sea trimestral o anual. Si $r_{i}$ es el coeficiente de correlación entre el indicador $i$ y la variable de referencia, el peso de cada uno de los indicadores en la definición del índice compuesto será

$$
w_{i}=\frac{r_{i}}{\sum_{i=1}^{n} r_{i}}
$$

donde $n$ será el número de indicadores utilizados.

El último método para asignar pesos se basa en el análisis de regresión entre la variable de referencia y los indicadores parcia- 
les, utilizando los coeficientes de regresión como la contribución de cada indicador parcial. Este método constaría de los siguientes pasos: en primer lugar se obtendría el coeficiente de correlación (ri) entre la señal extraída del indicador parcial seleccionado y la magnitud de referencia (lógicamente, deben tener la misma frecuencia, mensual, trimestral o anual). En segundo lugar, la obtención de las ponderaciones para cada uno de los indicadores como

$$
w_{i}=\frac{r_{i}}{\sum_{i=1}^{n} r_{i}}
$$

Por último, obtendríamos los valores del indicador sintético en cada momento de tiempo $t$,

$$
I_{t}=\sum_{i=1}^{n} w_{i} \cdot x_{i t}
$$

Basados en el análisis de la regresión también existen procedimientos conocidos como combinación de datos de alta y baja frecuencia porque -como ventaja fundamental- permiten la combinación de datos de baja frecuencia (que suele ser la que caracteriza a los indicadores de referencia) con información de alta frecuencia (que son los datos que habitualmente ofrecen los indicadores parciales), siempre bajo el supuesto de que la relación estimada con bajas frecuencias se mantiene cuando se utilizan altas frecuencias. El inconveniente de este método aparece por la habitual existencia de multicolinealidad en la regresión anual, introduciendo elementos de duda en la influencia de cada una de las señales anualizadas (estadísticos t no significativos), que aunque en teoría no invalidan el modelo a efectos predictivos, en la práctica sí es necesario rechazar el modelo por la habitual presencia de signos equivocados o valores poco probables en los coeficientes. Además, la consecución de nuevos datos suele provocar grandes oscilaciones en la estimación de los parámetros.

Dentro de este último método se encuadran los procedimientos de selección automática. La problemática es similar al caso anterior: se dispone de las señales como variables independientes y una macromagnitud como variable dependiente. No obstante, ahora se trata de elegir la mejor regresión que se pueda realizar utilizando sólo algunas señales como variables independientes (aquéllas que manifiestan una influencia significativa sobre el regresando y que son seleccionadas por el proceso). 
Este método sirve para seleccionar los regresores de forma automática (en el sentido de que sólo se atiende a criterios estadístico-econométricos). El uso de este tipo de procedimientos tiene la ventaja de que no es necesario estimar todas las ecuaciones posibles, el mismo nos proporciona la que considera la mejor regresión; por otro lado, facilitan un instrumento que es un buen predictor de una variable dependiente. Entre los inconvenientes destacan, además de los ya mencionados, el uso de data mining para la selección de indicadores parciales, de manera que sólo tenemos en cuenta criterios estadístico-econométricos en la selección y no incluye ninguna información de tipo económico.

\subsection{Metodología del National Burean of Economic Research $y$ del Bureau of Economic Analisis}

El Bureau of Economic Analysis (BEA), dependiente del Departamento de Comercio de los Estados Unidos, publica mensualmente en el Survey of Current Business un sistema de indicadores de la economía estadounidense y presenta un procedimiento para la elaboración de un índice. La metodología utilizada por estas dos instituciones es similar (aunque a continuación describimos el método usado por el BEA y señalamos las diferencias) y se compone de las siguientes fases (Green y Beckman, 1992).

1) Cálculo del cambio porcentual simétrico de cada indicador,

$$
c_{t, i}=200 \frac{x_{t, i}-x_{t-1, i}}{x_{t, i}+x_{t-1, i}}
$$

donde $x_{i}$ es el i-ésimo indicador parcial.

2) Estandarización de las series obtenidas en la etapa anterior para impedir que las oscilaciones de un indicador parcial dominen a las variaciones de los demás, es decir, se define $\mathrm{S}_{\mathrm{i}}$ como la media absoluta del porcentaje de cambios mensuales antes obtenido

$$
S_{t, i}=\frac{c_{t, i}}{\sum_{t-2}^{T} \frac{\left|c_{t, i}\right|}{T-1}}
$$

3) Asignación de ponderaciones a cada indicador parcial (Wi),

$$
\beta_{i}=\frac{1}{I S_{i}}
$$


donde $I$ es el número de indicadores parciales. Para garantizar que la suma de las ponderaciones sea unitaria se transforma en

$$
w_{i}=\frac{\beta_{i}}{\sum_{1}^{I} \beta_{i}}
$$

4) El porcentaje de cambio en un periodo del índice se define como

$$
r_{t}=\sum_{i=1}^{I} W_{i} x_{i t}+T R_{i}
$$

siendo $T R$ un factor de ajuste tendencial.

5) Por último, se calcula el índice sintético coincidente como

$$
I S_{t}=I S_{t-1} \frac{200+r_{t}}{200-r_{t}}
$$

donde se suele asignar un valor inicial de $I S_{0}=100$.

El método del National Bureau of Economic Research (NBER) difiere en la forma de estandarizar las oscilaciones en la etapa 2 y en cómo se asignan las ponderaciones. Además, el ajuste de la tendencia se produce una vez obtenido el índice mediante una media móvil de 75 términos denominada Phase Average Trend. Es importante señalar que estos indicadores no pretenden medir el ritmo de variación económica sino detectar los puntos de giro con la máxima rapidez y eficacia, ésta es la principal diferencia con respecto a los métodos basados en el análisis de la regresión.

\subsection{Análisis multivariante}

El análisis de componentes principales y el análisis factorial son los métodos más usados en la construcción de indicadores sintéticos. Ambos son procedimientos diseñados para el tratamiento de grandes masas de datos, transformando las variables originales -indicadores parciales- en otras -componentes principalesque no están correlacionadas entre sí. De este modo se puede proceder a la selección de información, eliminando las componentes que no proporcionan información significativa. Se trata de aplicar la técnica de los componentes principales a los crecimientos interanuales tipificados de las señales, es decir, se toman diferencias estacionales del logaritmo del indicador para a continuación estandarizar el valor de la serie. 
Sea $Y_{i t}=\nabla_{4} \ln X_{i t}$ para datos trimestrales

$$
Z_{i t}=\frac{X_{i t}-\mu\left(X_{i t}\right)}{\sigma\left(X_{i t}\right)}
$$

A continuación se especifica el modelo unifactorial, $Z_{t}=\Gamma f_{t}+\varepsilon_{t}$, donde $Z_{t}$ es el vector de observaciones de los indicadores (I) que van a formar el indicador sintético, $G$ es el vector de cargas y $f$ es el factor común no observable. El vector $\mathrm{e}_{\mathrm{t}}$ recoge los factores específicos de cada indicador. Lo que se pretende es obtener una estimación del factor $f$ no observable que está describiendo el comportamiento conjunto de las series, considerando las hipótesis de,

* Normalidad tipificada del factor común, $f_{t} \sim N(0,1)$.

* Los factores específicos son heterocedásticos, $E\left(\varepsilon_{t}\right)=0, E\left(\varepsilon_{t} \varepsilon_{t}^{\prime}\right)=\Omega$, diagonal .

* Los factores comunes y los específicos son ortogonales, $E\left(f_{t}^{\prime} \varepsilon_{t}\right)=0$.

Con estos supuestos podemos estimar $f_{\mathrm{t}}$ por mínimos cuadrados generalizados para obtener

$$
f_{t}^{e}=\left(\Gamma^{\prime} \Omega^{-1} \Gamma\right)^{-1}\left(\Gamma^{\prime} \Omega^{-1} Z_{t}\right)=W Z_{t}=\sum_{i} w_{i} Z_{i t}
$$

Es decir, $f$ es una combinación lineal de los indicadores parciales. Se suelen presentar los pesos normalizados a suma uno,

$$
f_{t}^{e}=\sum_{i} u_{i} Z_{i t}, \quad \text { con } u_{i}=\frac{w_{i}}{\sum_{i} w_{i}}
$$

Las componentes se ponderan en función del porcentaje de varianza que explican, obteniéndose el indicador sintético como suma de dichos componentes ponderados $(u)$. La principal ventaja del análisis multivariante para la elaboración de índices sintéticos de actividad económica es que al tratarse de una técnica de reducción de la dimensión, permite resumir en un número reducido de factores o dimensiones la información contenida en un conjunto elevado de indicadores parciales. 


\subsection{Procedimiento simple de Niemira y Klein (NIEM)}

El índice sintético de Niemira y Klein (1994) se obtiene con la suma de los crecimientos de la señal de cada indicador parcial, pero teniendo en cuenta la importancia y volatilidad de cada uno de esos indicadores. En definitiva, se trata de aplicar la fórmula

$$
N I E M=\sum_{i}^{n} w_{i} \cdot s_{i} \cdot T_{i}
$$

donde $T_{i}$ denota la tasa de crecimiento en tantos porcentuales del indicador $i$-ésimo, siendo $i=1, \ldots, n$ el número de indicadores parciales, wi es el peso o ponderación que se aplica a cada indicador parcial, $s_{\mathrm{i}}$ es el elemento de ajuste de la volatilidad del indicador parcial, una amplitud estandarizada para todos los indicadores parciales con el fin de minimizar la influencia de un indicador individual muy volátil sobre el indicador sintético. El indicador se elabora del siguiente modo:

1. Determinación de las ponderaciones.

2. Determinación de la volatilidad.

3. Determinación del peso de los indicadores parciales.

4. Cálculo del indice compuesto,

$$
\text { NIEM }=\sum_{i=1}^{n} p_{i} \cdot \text { Indicador }_{i}
$$

\subsection{Procedimiento basado en Fernández (1991)}

Este procedimiento es una adaptación del esquema de agregación seguido por el NBER. A continuación explicamos este procedimiento atendiendo a la exposición recogida en Fernández (1991), pero adecuado a la construcción de un indicador sintético de crecimiento de la actividad económica.

a) Se calculan las tasas de variación trimestrales de cada señal de ciclo tendencia,

$$
T_{1}^{1}\left(x_{t}\right)=\frac{x_{t}-x_{t-1}}{x_{t-1}}
$$

b) Se estandarizan las tasas de crecimiento de las señales de ciclo-tendencia $\left[T_{1}^{1}\left(x_{i t}\right)\right]$ para que tengan una amplitud co- 
mún, es decir, dividir cada una de ellas entre la media aritmética de sus valores absolutos,

$$
v_{i t}=\frac{T_{1}^{1}\left(X_{i t}\right)}{T_{1}^{1}\left(X_{i t}\right)} ; \quad t=1, \ldots, T
$$

donde

$$
\overline{T_{1}^{1}}\left(X_{i t}\right)=\frac{1}{T} \sum_{t=1}^{T}\left|T_{1}^{1}\left(X_{i t}\right)\right|, \quad \forall i
$$

c) Se obtiene la serie suma de las series estandarizadas,

$$
w_{t}=\sum_{t=1}^{n} v_{i t}
$$

d) Se obtiene un indicador que permitirá llevar a cabo el seguimiento del crecimiento interanual del VAB trimestral,

$$
\left(T_{1}^{4}\left(V A B_{t}\right)=\frac{V A B_{t}-V A B_{t-4}}{V A B_{t-4}}\right)
$$

Para lo cual se procede del siguiente modo:

$$
\text { Fernández }=\sum_{j=-2}^{1} w_{t+j}
$$

\subsection{Procedimiento basado en la distancia $P_{2}$}

La aplicación original del indicador con base en la distancia $\mathrm{P}_{2}$ va dirigida al campo de la medición del bienestar social. A continuación se presenta de manera resumida el indicador Zarzosa (1992). Sea el vector de estado de las señales de ciclo tendencia extraídas de los indicadores parciales del momento de tiempo $t=1, \ldots, \mathrm{T}, \mathrm{X}_{\mathrm{t}}$ $=\left(\mathrm{x}_{\mathrm{t} 1}, \mathrm{x}_{\mathrm{t} 2}, \ldots, \mathrm{x}_{\mathrm{tn}}\right)$, de manera que $\mathrm{x}_{\mathrm{ti}}(i=1, \ldots, n)$ es el valor que toma la señal de ciclo-tendencia del indicador parcial en el momento de tiempo $t$ (en adelante se denota como señal $i$ ).

Este vector de estados se quiere comparar con un vector de frecuencia. Sea X. el vector base de referencia, donde X. $=\left(x_{._{1}}\right.$ $\left.\mathrm{x}_{{ }_{2}}, \ldots, \mathrm{x}_{\cdot_{\mathrm{T}}}\right)$. De esta forma, $\mathrm{x}_{{ }_{\mathrm{t}}}$ es el estado base de referencia en el momento $t$, un estado ideal para las señales extraídas de cada uno de los indicadores parciales de referencia con respecto al cual se realiza la comparación. En este caso, la base de referencia está constituida por el vector que toma para cada momento $t$ el valor mínimo de cada una de las señales cuya información se pretende agregar. 
De esta forma, el indicador sintético de distancia $\mathrm{P}_{2}$ se define para cada momento como (Zarzosa, 1992),

$$
D P_{2}(t)=\sum_{i=1}^{n} \frac{d_{i}}{\sigma_{i}}\left(1-R_{i-1, i-2, \ldots 1}^{2}\right) \text { donde } R_{1}^{2}=0
$$

Las condiciones o propiedades exigibles por la función matemática que genera al indicador sintético son las siguientes: existencia y determinación, monotonía, unicidad, homogeneidad, transitividad, exhaustividad, aditividad e invarianza respecto de la base de referencia (Zarzosa, 1992).

\subsection{Construcción de indicadores en espacios de estados}

Como ya se expuso, la obtención de un indicador sintético como media ponderada de indicadores parciales se basa, además de en la selección de éstos, en la determinación de los pesos relativos que tiene cada uno de ellos. Si la selección está bien realizada, es de esperar que los indicadores parciales no presenten un comportamiento independiente, sino que estén influidos por la evolución general de la macromagnitud estudiada. La estimación de este factor de evolución general es el objetivo de los indicadores sintéticos, abordando ésta mediante la combinación lineal ponderada de los indicadores parciales.

Por ello, se puede interpretar un indicador sintético como un factor común al comportamiento del conjunto de indicadores parciales, cuya evolución condiciona, con cierta intensidad, la de estos últimos. Dentro de este marco conceptual es donde presenta gran interés la modelización conjunta de los indicadores en espacio de estados. Recordando la expresión de la representación innovacional de una serie múltiple centrada (Mondéjar, 2007),

$$
\begin{aligned}
& X_{t+1}=F X_{t}+G \varepsilon_{t} \\
& Y_{t}=\mu+H X_{t}+\varepsilon_{t} \quad t \in Z
\end{aligned}
$$

donde $Y$ es el vector de indicadores parciales y $1 / 4$ es su vector de medias, la estructura básica del modelo supone la existencia de un vector de estado, $X_{t}$, que actúa en cada instante como estadístico suficiente para la dinámica del sistema, por lo que puede identificarse como el factor de evolución general o indicador sintético cuya estimación se pretende. 
Tabla 1

Indicador sintético sectorial: industria

\begin{tabular}{ll}
\hline \multicolumn{1}{c}{ Indicador } & Frecuencia \\
\hline Activos en industria & Trimestral \\
Ocupados en industria & Trimestral \\
Paro registrado en industria & Mensual \\
Producción bruta de electricidad & Mensual \\
Índice de producción industrial & Mensual \\
Consumo de energía industria & Mensual \\
Consumo de energía para uso industrial & Mensual \\
Matriculación de vehículos industriales & Mensual \\
\hline
\end{tabular}

Fuente: Elaboración propia.

En este caso, la metodología de espacio de estados permite una estimación eficiente de dicho indicador, ${ }^{2}$ sin necesidad de recurrir a una media ponderada de los indicadores parciales, sino modelizando la correlación entre el indicador sintético y cada uno de los parciales, reflejada en la matriz H. Además, presenta otras ventajas.

- Como señala la ecuación de transición, el indicador sintético presenta una evolución markoviana corregida por el efecto de las innovaciones de los indicadores parciales a través de la matriz G.

- El estado, identificado con el indicador sintético, refleja la evolución subyacente del conjunto de indicadores parciales, ya que la mejor predicción del vector de observaciones para el siguiente instante temporal sería

$$
\hat{Y}_{t+1 \mid t}=\mu+H X_{t}
$$

Por ello, el estado podría interpretarse como la componente tendencial ${ }^{3}$ del conjunto de indicadores parciales.

- La estructura de la ecuación de observación permite extraer la componente innovacional de cada indicador parcial. Ésta se introduce en la evolución del indicador sintético a través de la matriz $G$, lo que permite que éste responda rápidamente a novedades en los indicadores parciales y, además, cuantificar la intensidad relativa con la

${ }^{2}$ Este hecho es resultado de que el filtrado de Kalman (1960) no es más que la estimación insesgada y de varianza mínima de los estados.

${ }^{3}$ En el sentido de que sería la evolución esperada si no hubiese innovaciones dentro de los indicadores parciales. 
Gráfica I Indicadores de industria

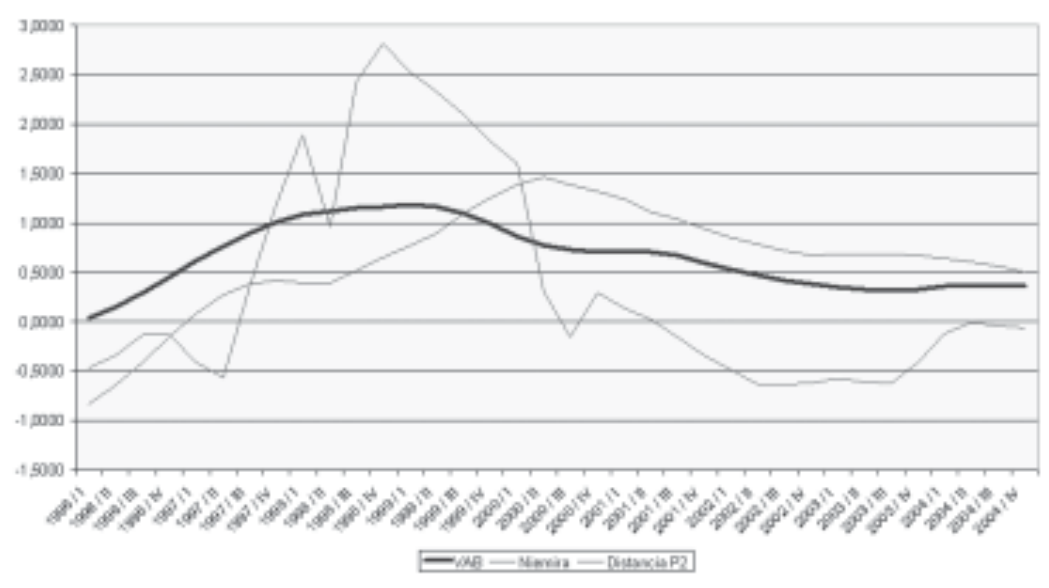

Fuente: Elaboración propia.

que cada uno de estos últimos modifica la evolución del indicador sintético.

- La representación en espacio de estados permite obtener rápidamente la función de impulso-respuesta mediante las matrices del modelo como

$$
Z_{k}=H F^{K-1} G, \quad K \in \text { ù }
$$

que permite valorar el efecto de cada indicador parcial sobre el sintético.

Por todo ello, la utilización de la metodología de espacio de estados puede ser una alternativa fructífera para obtener un indicador sintético.

\section{Trabajo empírico}

En el marco del análisis económico regional, un foco de atención constante es el análisis coyuntural, con el que se pretende conocer lo antes posible la situación y el ritmo de crecimiento de la actividad de la región y sus posibles causas. La utilización de los indicadores sintéticos para este fin no es reciente; la publicación de un sistema de indicadores por el BEA sirvió de base para la construcción de indicadores sintéticos del NBER. Desde entonces 
Gráfica II Indicadores de industria

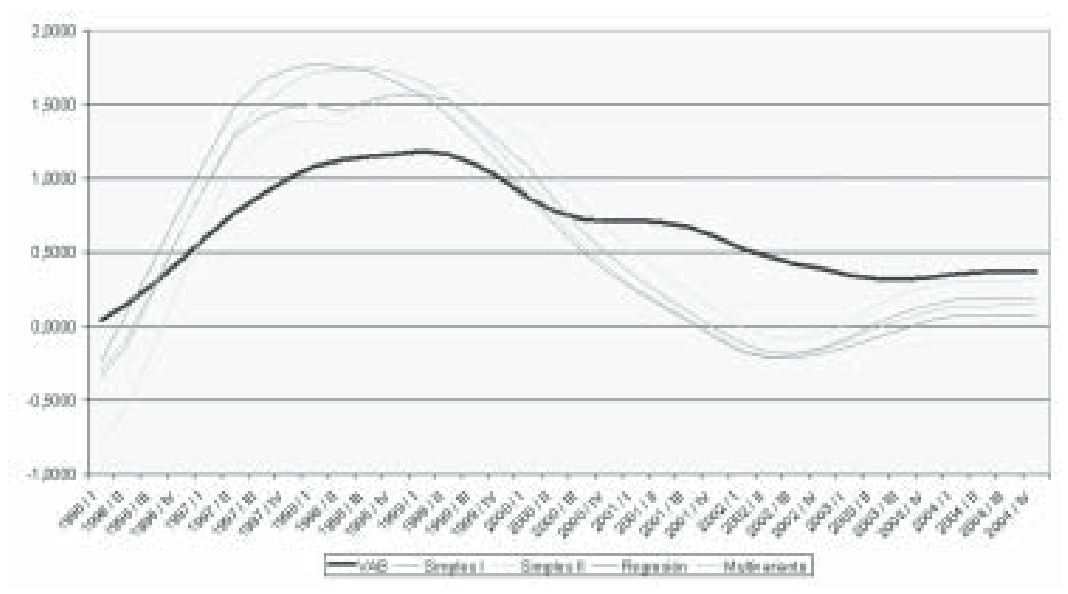

Fuente: Elaboración propia.

han proliferado en este campo las aportaciones metodológicas y sus aplicaciones.

La mayor dificultad que podemos encontrar en este sentido y que constituye, a su vez, requisito imprescindible para construir un indicador sintético regional de coyuntura, es la necesidad de disponer de una amplia base de datos de índole regional y de periodicidad mensual o trimestral (indicadores parciales) que permitan captar las fluctuaciones periódicas de corto plazo de la economía objeto de análisis.

En primer lugar, en la tabla 1 enumeramos los indicadores parciales seleccionados por su disponibilidad, periodicidad, desfase, calidad, etc., todos ellos referidos al sector industrial dentro del ámbito regional de Castilla-La Mancha.

Una vez seleccionados los indicadores parciales con base en criterios de disponibilidad, frecuencia, volatilidad, significación económica, etc., procedemos a extraer la señal relevante de los mismos. Con ésta, hacemos la agregación de los mismos, con base en alguno de los diferentes criterios referidos en el apartado 1, con la excepción de la metodología propuesta por el NBER y el procedimiento basado en Fernández, debido a que el objetivo de estos métodos de agregación de indicadores es detectar los puntos de giro de la economía, en lugar de realizar un seguimiento de la evolución de la misma.

Una vez que se obtuvieron los indicadores sintéticos, se debe proceder a seleccionar los indicadores resultantes de cada méto- 


\section{Gráfica III}

Indicadores de industria

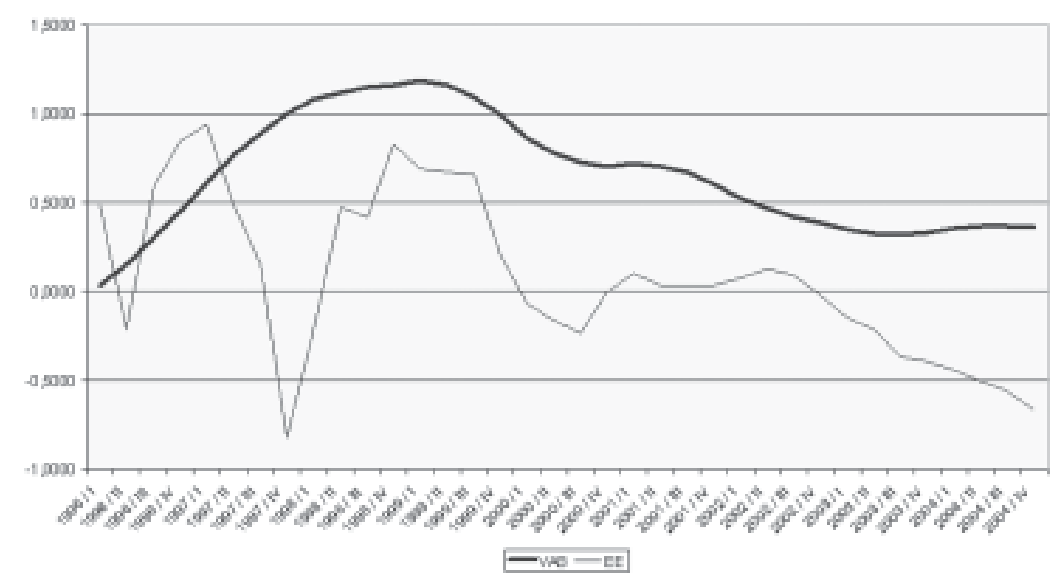

Fuente: Elaboración propia.

do de agregación, para lo cual es necesario tener en cuenta los siguientes criterios:

- Volatilidad de los indicadores sintéticos para evitar demasiadas fluctuaciones o que no detecten los cambios existentes.

- Adecuación de las series disponibles. La longitud de las mismas y la frecuencia son dos de los factores condicionantes $a$ priori a la hora de elegir los métodos de agregación.

- Correlación con las variables de referencia. Debemos medir el grado de correlación entre los indicadores sintéticos y la variable de referencia.

- Fechado de los indicadores sintéticos. Es deseable que el indicador sintético sea adelantado.

- Estructura del retardo. La estructura del mismo condiciona los resultados obtenidos.

- Disponibilidad de los datos. La rápida disponibilidad de los datos condiciona la utilización de los indicadores sintéticos.

El análisis de la correlación y el retardo temporal constituyen las dos opciones más importantes en términos de validación, si bien el resto supone más bien criterios de partida.

En las gráficas I, II, y III de indicadores de industria observamos la aplicación de algunos de los criterios más extendidos a un caso real de economía regional, concretamente el sector industrial de Castilla-La Mancha, agrupados por criterios de selec- 
ción de indicadores sintéticos. Así, la gráfica I muestra los métodos de agregación con resultados menos satisfactorios. La distancia $\mathrm{P}_{2}$, concebida inicialmente como una medida de bienestar, presenta un alto grado de volatilidad, con una relativa coincidencia cíclica con el valor añadido bruto (VAB) y un escaso grado de correlación contemporánea. Por su parte, el indicador obtenido por el procedimiento de Niemira y Klein presenta mayor grado de correlación y menor volatilidad; sin embargo, es un indicador retrasado respecto de la variable objetivo; lo que invalida parcialmente que lo utilicen los agentes económicos más interesados en anticipar las fluctuaciones del sector.

En la gráfica II se muestra un conjunto de indicadores sintéticos con una mayor volatilidad que la variable original, una evidente coincidencia cíclica y alto grado de correlación. Este tipo de indicadores constituyen la alternativa más frecuente para analizar la coyuntura económica tanto por su sencillez en el cálculo como por su alto grado de correlación contemporánea. Sin embargo, su capacidad predictiva es limitada ya que no anticipa el comportamiento cíclico de la variable original.

Por último, en la gráfica III se representa el indicador sintético en espacio de estados (EE). Por varios motivos, el mencionado indicador constituye una alternativa para los métodos tradicionales.

- En primer lugar, es el único indicador que adelanta los puntos de giro de la producción industrial en Castilla-La Mancha. Esta característica es cada vez más apreciada por los agentes económicos que necesitan la anticipación del posible escenario económico para la planificación de sus decisiones de financiamiento, producción e inversión.

- También presenta un mayor grado de volatilidad respecto de las alternativas de la gráfica II y un grado de correlación contemporánea ligeramente inferior. Sin embargo, este último hecho se debe sobre todo al retardo entre las dos variables. Así, si se considera la correlación corregida por periodo se obtienen buenos resultados.

Otras aplicaciones empíricas más profundas, con resultados similares, se pueden consultar en Cabrer et al. (2001), Mondéjar (2007), Mondéjar y Alfaro (2002) y Pavía (2001). 


\section{Conclusiones}

Por todos es conocida la preocupación de los diferentes agentes económicos, especialmente las entidades nacionales y supranacionales, por anticipar las posibles fluctuaciones económicas que condicionan su toma de decisiones. Dentro del análisis coyuntural, la aplicación de las diferentes técnicas nos lleva a resultados más o menos costosos debido a su complejidad o la disponibilidad de los datos. En el presente trabajo se pretendió desarrollar los diferentes métodos de agregación para la construcción de indicadores sintéticos de actividad regionales, con el fin de obtener resultados de forma rápida y sencilla, pero con el mínimo error posible.

El crecimiento del interés por el tema dentro del campo de la economía aplicada se pone de manifiesto en la proliferación de trabajos en los últimos años con el único objetivo de predecir los cambios regionales. En España resultan de especial interés este tipo de estudios por la escasez de información estadística de carácter coyuntural en el ámbito regional.

Los diversos métodos de agregación de los indicadores parciales constituyen la parte más importante del trabajo. Además de una exposición de éstos, se trata de mostrar los métodos más utilizados en la predicción de macromagnitudes regionales. La utilización de un único método de agregación y su aplicación al ámbito regional puede ser bastante compleja, ya que éstos no son excluyentes entre sí.

A los métodos de agregación tradicionales se ha incluido uno nuevo. La metodología en espacio de estados permite una estimación eficiente de dicho indicador, sin necesidad de recurrir a una media ponderada de los indicadores parciales, sino modelizando la correlación entre el indicador sintético y cada uno de los parciales, reflejada en la matriz $\mathrm{H}$.

En la metodología en espacio de estados, las propiedades de controlabilidad y observabilidad garantizan, bajo supuestos poco restrictivos, la minimalidad de la representación. Esta propiedad presenta dos grandes ventajas: por un lado, si el algoritmo de especificación de un modelo en espacio de estados proporciona la controlabilidad y observabilidad del sistema, se tiene garantizada la menor dimensión para el vector de estados que recoge la dinámica del proceso; por otro, si se trabaja con modelos VARMA se puede obtener una representación canónica alternativa mediante la reexpresión del modelo estimado en espacio de esta- 
dos. De esta forma conseguimos ajustar el modelo con un número de parámetros involucrados inferior a los necesarios en una modelización VARMA y, además, podemos afirmar que esta representación cumple el requisito de minimalidad. Es por ello que los indicadores en espacio de estados constituyen una alternativa para los métodos tradicionales debido a que estos indicadores sintéticos en espacio de estados registran y reproducen de manera inmediata cualquier cambio en los indicadores parciales, mientras que los métodos tradicionales no logran recoger rápidamente las alteraciones registradas en los indicadores parciales. Por tanto, los mencionados indicadores pueden constituir un indicador sintético adelantado, de vital importancia informativa para los agentes económicos interesados.

Los métodos de agregación basados en regresión o en técnicas multivariantes destacan por su sencillez de cálculo y buenos resultados de correlación, pero no son capaces de adelantar las fluctuaciones de la variable de referencia. Actualmente, los agentes económicos están cada vez más interesados en anticipar el comportamiento de la economía real, por lo que existe un interés creciente por utilizar técnicas que mitiguen esta carencia.

\section{Bibliografía}

Cabrer, Bernardí, Javier de Castro y José Manuel Pavía (2001), "Indicadores económicos regionales y su problemática: una visión de síntesis”, en Bernardí Cabrer Borrás (ed.), Análisis regional. El proyecto Hispalink, Mundiprensa, Madrid, España, pp. 259-277.

Fernández, Francisco (1991), “Indicadores sintéticos de aceleraciones y desaceleraciones en la actividad económica”, $R e$ vista Española de Economía, 8(1), Instituto de Estudios Fiscales, Madrid, España, pp. 125-156.

Green, George B. y Barry A. Beckman (1992), “The composite index of coincident indicators and alternative coincident indexes", Survey of Current Business, 72, Department of Commerce, Washington, D.C., pp. 42-45.

Kalman, Rudolf Emil (1960), “A New Approach to Linear Filtering and Prediction Problems", Journal of Basic Enginee- 
ring, Transactions of ASME, 82, Transactions of ASME, Nueva York, Estados Unidos, pp. 35-45.

Maravall, Agustín (1989), "La extracción de señales y análisis de coyuntura", Revista Española de Economía, 6(1-2), Instituto de Estudios Fiscales, Madrid, España, pp. 7-56.

Mondéjar Jiménez, José (2007), "Análisis cuantitativo de la coyuntura económica. Una aplicación de la representación en espacio de estados de series temporales múltiples", tesis doctoral, Universidad de Castilla-La Mancha, España, $524 \mathrm{pp}$.

Mondéjar Jiménez, José y José Luis Alfaro Navarro (2002), “Construcción de indicadores sintéticos de coyuntura económica. El caso de Castilla-La Mancha”, xxvIII Reunión de Estudios Regionales, Universidad de Murcia, España, 16 pp.

Niemira, Michael P. y Philip A. Klein (1994), Foresting Financial and Economic Cycle, Nueva York, John Wiley \& Sons, Estados Unidos, 544 pp.

Pavía, José Manuel (2001), "Una aproximación a la elaboración de indicadores sintéticos: el caso de la Comunidad Valenciana”, en Bernardí Cabrer Borrás (ed.), Análisis regional. El proyecto Hispalink, Mundiprensa, Madrid, España, pp. 291-301.

Pons-Novell, Jordi (1995), "Un sistema d'indicadors cíclics per a l'economia catalana, Un instrument per a l'anàlisi conjuntural", tesis doctoral, Universitat de Barcelona, España, $227 \mathrm{pp}$.

Zarzosa-Espina, Pilar (1992), "Aproximación a la medición del bienestar social, estudio de la idoneidad del indicador sintético 'Distancia P2'”, tesis doctoral, Universidad de Valladolid, España, 248 pp.

Recibido: 9 de octubre de 2006. Reenviado: 23 de enero de 2007. Aceptado: 22 de mayo de 2007. 
José Mondéjar Jiménez. Es doctor europeo en economía por la Universidad de Castilla-La Mancha. Recibió el premio a la mejor tesis doctoral "Castilla-La Mancha, Región de Europa” que otorga el Gobierno de Castilla-La Mancha. Actualmente es profesor titular del área de estadística en el Departamento de Economía Política y Hacienda Pública, Estadística Económica y Empresarial y Política Económica de la Facultad de Ciencias Sociales de Cuenca de la Universidad de Castilla-La Mancha. Sus principales líneas de investigación son: indicadores sintéticos, herramientas estadísticas para el análisis de la coyuntura económica, predicción de macromagnitudes, desarrollo rural y turismo. $\mathrm{Ha}$ realizado estancias de investigación en otras universidades españolas y europeas y participa en varios proyectos de investigación. Es autor de diversas ponencias y comunicaciones aceptadas y presentadas en congresos nacionales e internacionales, así como autor de artículos publicados en revistas científicas, libros y capítulos en libros.

Manuel Vargas Vargas. Es doctor en economía por la Universidad de Castilla-La Mancha. Actualmente es profesor titular del área de estadística en el Departamento de Economía Política y Hacienda Pública, Estadística Económica y Empresarial y Política Económica de la Facultad de Ciencias Sociales de Cuenca de la Universidad de Castilla-La Mancha. Sus principales líneas de investigación son: series temporales múltiples, herramientas estadísticas para el análisis de la coyuntura económica, predicción de macromagnitudes, espacio de estados y desarrollo rural. Participa en diversos proyectos de investigación. Es autor de diversas ponencias y comunicaciones aceptadas y presentadas en congresos nacionales e internacionales, así como autor de artículos publicados en revistas científicas y capítulos en libros. 